\title{
Endoscopic Ultrasound-Guided Mixture of Glue and Lipiodol Injection in a Pseudoaneurysm Arising from the Artery of Drummond
}

\author{
Pankaj N. Desai ${ }^{1}$ Mayank Kabrawala ${ }^{1}$ Subhash Nandwani ${ }^{1} \quad$ Rajiv Mehta $^{1} \quad$ Chintan Patel ${ }^{1}$ \\ Parika Kalra ${ }^{1}$ Ritesh Prajapati ${ }^{1} \quad$ Nisharg Patel ${ }^{1}$
}

\author{
${ }^{1}$ Department of Endoscopy and EUS, Surat Institute of Digestive \\ Sciences, Surat, Gujarat, India
}

J Digest Endosc 2019;10:197-200

\section{Introduction}

Upper gastrointestinal bleeding most common causes are peptic ulcer, esophagitis, portal hypertension-related conditions, and malignancy. We are presenting a case of pseudoaneurysm arising from artery of Drummond as malena.

\section{Case}

A 63-year-old male patient presented with abdominal pain, nausea, and vomiting for 3 days and profuse malena for 1 day. On evaluation his hemoglobin dropped from 13.6 to $10.5 \mathrm{~g} / \mathrm{dL}$ with postural hypotension.

An upper gastrointestinal endoscopy was performed and it did not reveal any source of bleeding. Computed tomography (CT) of abdomen with angiography revealed a pseudocyst arising in the region of the tail of the pancreas measuring around $25 \times 24 \mathrm{~mm}$ and a pseudoaneurysm in it with bleeding. The pseudoaneurysm was arising from the artery of Drummond ( - Fig. 1). Interventional radiologist could not

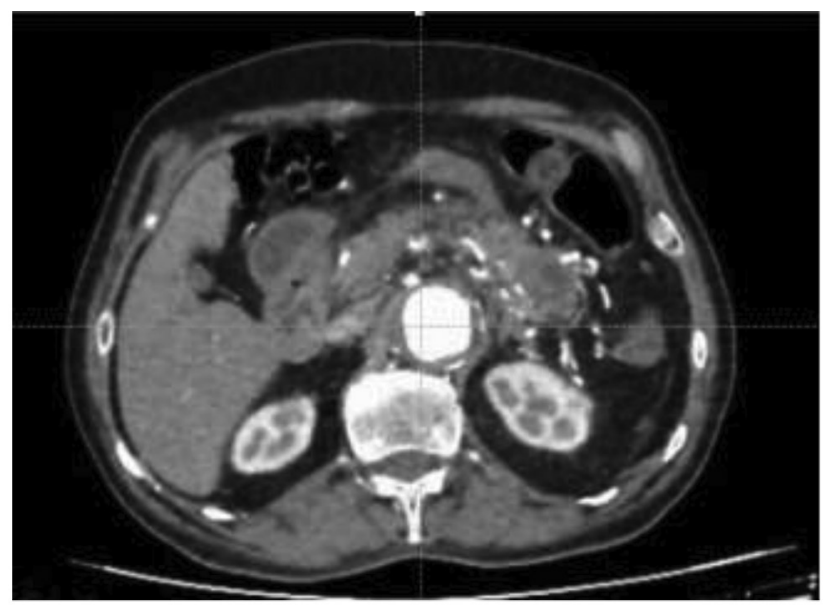

Fig. 1 CT angiography showing pseudoaneurysm was arising from the artery of Drummond. CT, Computed tomography.
Address for correspondence Dr Pankaj N. Desai, MS, FASGE, FAIGE, SIDS Hospital, Surat, Gujarat, India (e-mail: drp_desai@hotmail.com).

do the intervention due to the distant nature of origin of the pseudoaneurysm. Therefore, it was decided to approach by endoscopic ultrasound.

Endoscopic ultrasound revealed the pseudocyst in the tail and the pseudoaneurysm in it (-Fig. 2). The pseudoaneurysm was confirmed with Doppler flow and punctured with a 22-guage needle. Mixture of $1 \mathrm{~mL}$ glue with $3 \mathrm{~mL}$ lipiodol was injected ( - Fig. 3 ). A reflux of the glue into the feeding vessel was observed and we made sure that the neck of the pseudoaneurysm completely blocked ( - Fig. 4). The Doppler signal was lost at the end of the procedure suggestive of complete obliteration of the pseudoaneurysm ( - Fig. 5). CT abdomen done in the evening the same day revealed complete obliteration of the pseudoaneurysm. After procedure patient's hemoglobin remained stable. There was no evidence of any mesenteric ischemia. The patient was discharge on the third day of postprocedure. A repeat CT scan at 6 months follow-up revealed persistent blockage of the pseudoaneurysm.

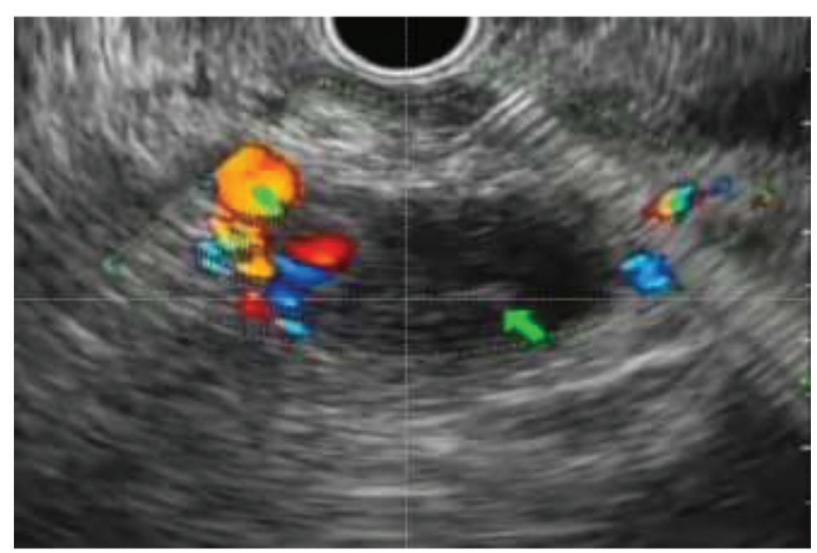

Fig. 2 EUS revealed the pseudocyst in the tail and the pseudoaneurysm in it. EUS, Endoscopic ultrasound.
License terms

(ㅇ)(1) $\Theta \circledast$ 


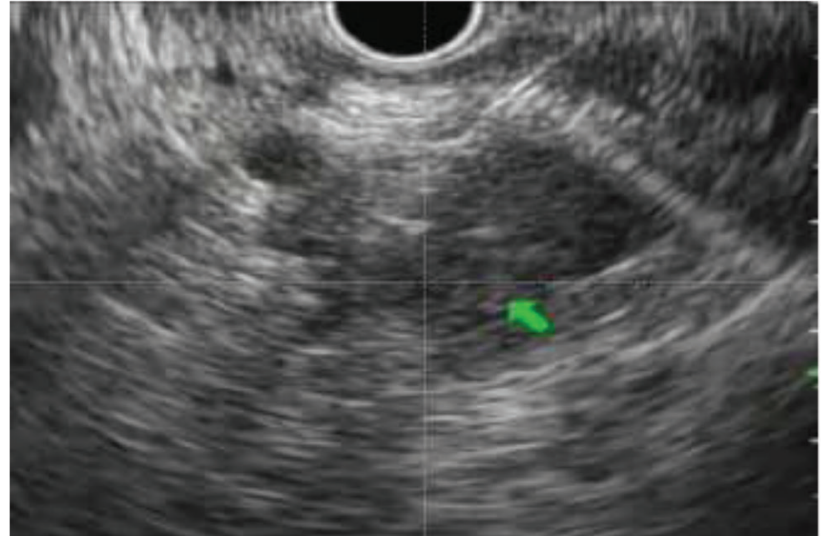

Fig. 3 Mixture of $1 \mathrm{~mL}$ glue with $3 \mathrm{~mL}$ lipiodol was injected into pseudoaneurysm.

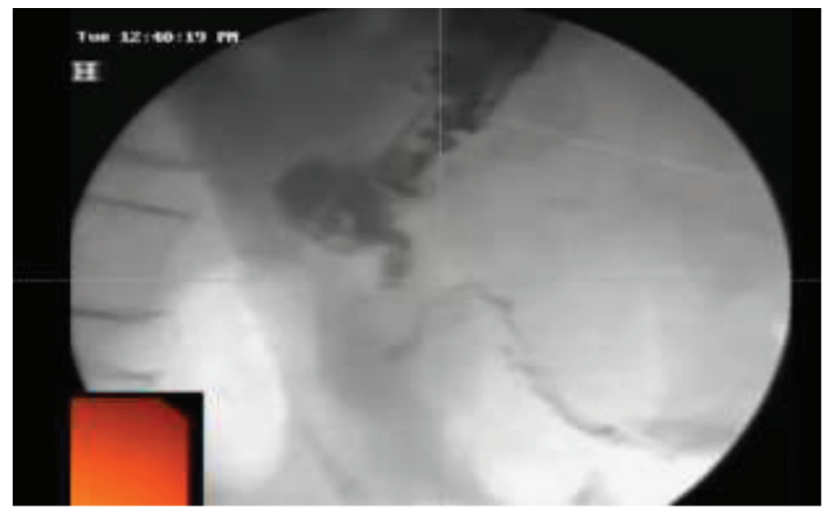

Fig. 4 Fluoroscopic image showing reflux of the glue into the feeding vessel and complete blockage of the neck of the pseudoaneurysm.

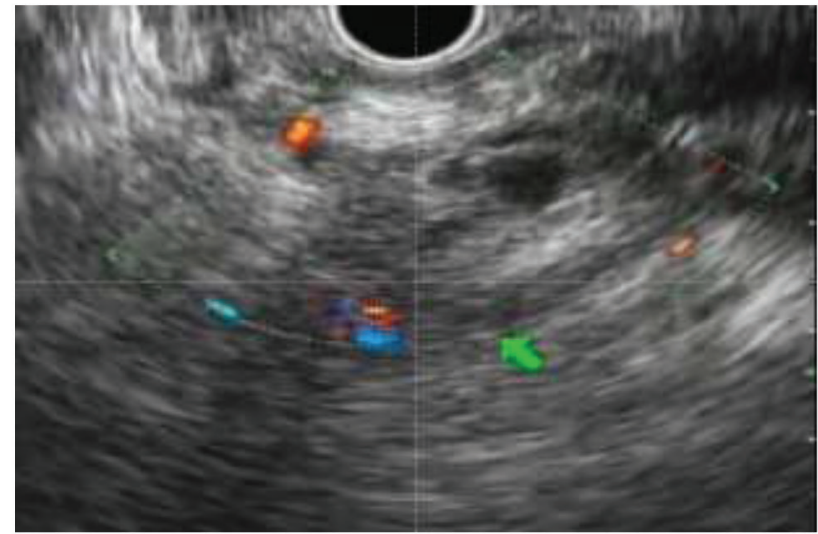

Fig. 5 EUS doppler signal was lost at the end of the procedure suggestive of complete obliteration of the pseudoaneurysm. EUS, Endoscopic ultrasound.

\section{Discussion}

The marginal artery of Drummond, also known as the marginal artery of the colon, is a continuous arterial circle or arcade along the inner border of the colon formed by the anastomoses of the terminal branches of the superior mesenteric artery and inferior mesenteric artery ( - Fig. 6). The marginal artery of Drummond is clinically important because it serves as a collateral supply to the colon when there is a blockage in one of the mesenteric vessels.

Injection of undiluted glue frequently leads to needle blockade and failure of therapy. This occurs due to reflux of blood in the needle. Undiluted glue has to be injected in short boluses with multiple needle punctures till complete obliteration of pseudoaneurysm.

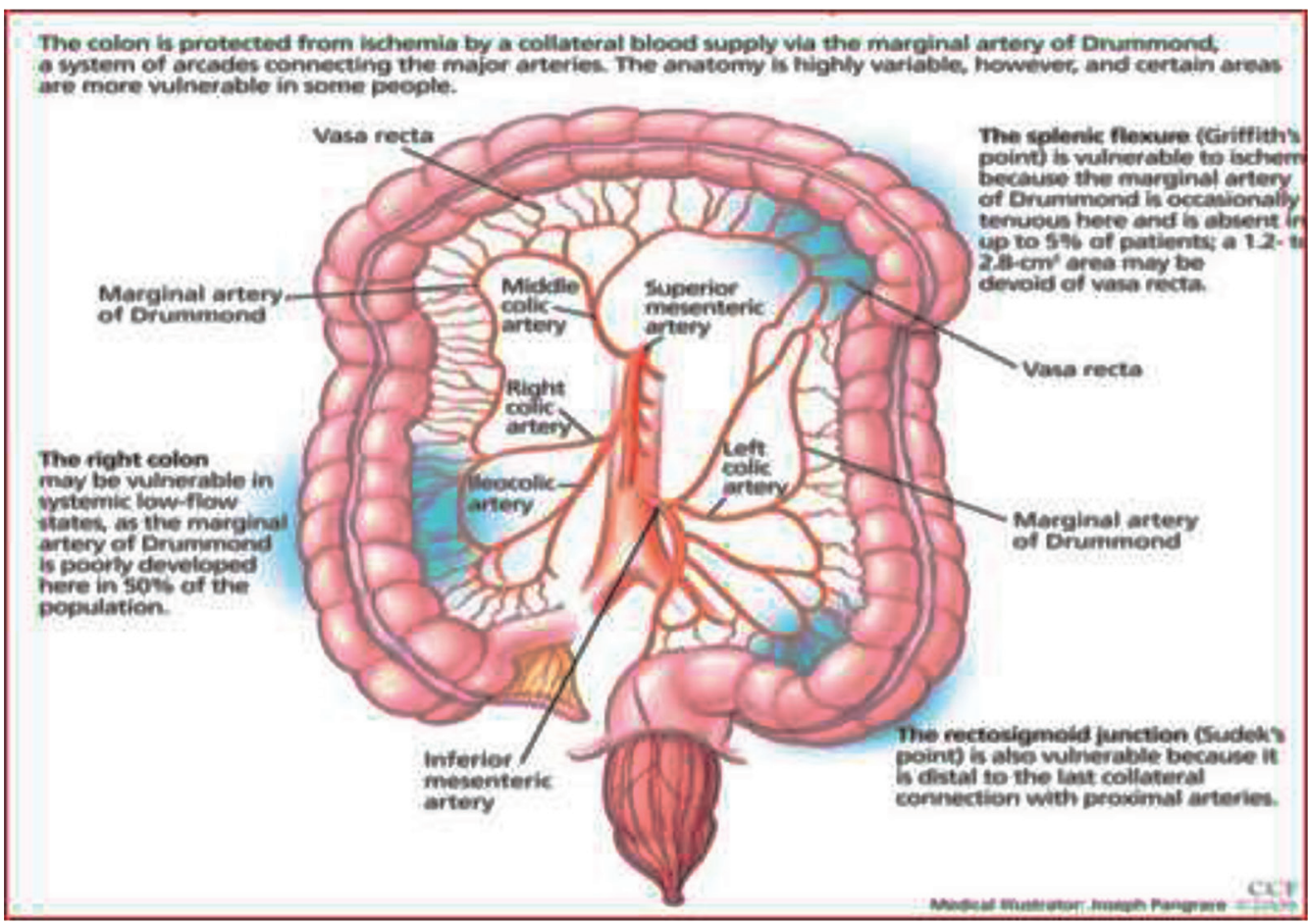

Fig. 6 Marginal artery of Drummond. 
Dilution of glue with lipiodol achieves two objectives:

1. It prevents needle blockade.

2. Allows steady glue injection till complete obliteration of pseudoaneurysm and hence avoid multiple punctures.

However, this may come at the cost of increased chances of glue embolization as seen in this case where there was spillage of glue in feeding vessel. But this spillage is an additional advantage as blocking of feeding vessel. This is in accordance to the laid down principles of interventional radiology.

Conclusions from one study on percutaneous glue embolization as a primary treatment for visceral pseudoaneurysms shows:

1. Endpoint of glue injection was considered when there was complete filling of the sac and reflux was seen in the feeding vessel.

2. A disadvantage is reflux into the parent vessel which can cause ischemia.

3. We deliberately tried to cause reflux of glue into the neck and beyond to cause complete obliteration of the vessel.

Primary glue injection mixed with lipiodol using EUS guidance has not been studied extensively. So we used guidance from the principles of interventional radiology.

\section{Take Home Message}

EUS-guided mixture of glue and lipiodol injection of peripancreatic pseudoaneurysm is feasible.

Addition of lipiodol allows controlled delivery of glue and avoids needle blockade.

Fluoroscopic visualization due to lipiodol acts as additional aid for procedure.

Understanding the anatomy of the feeding vessel before procedure is important to avoidinjecting the mixture of glue and lipiodol into an end artery and which may cause ischemia.

\section{Video 1}

EUS guided glue injection of pseudoaneurysm. Online content including video sequences viewable at: https:// www.thieme-connect.com/products/ejournals/html/ 10.1055/s-0039-3401964.

\section{Conflict of Interest}

None declared. 